\title{
Microbiota Analysis of Biofilms on Experimental Abutments Mimicking Dental Implants: An In Vivo Model
}

Berta Cortés-Acha, ${ }^{*}$ Rui Figueiredo, ${ }^{\star \dagger}$ Ramón Seminago, ${ }^{\ddagger}$ Francisco J. Roig,, Carlos Llorens, ${ }^{\ddagger}$ and Eduard Valmaseda-Castellón*†

Background: The microbiota colonizing dental implants has been said to be similar to the microbiome surrounding teeth. In the absence of inflammation, a biofilm with pathologic bacteria can cover implant surfaces exposed to the oral cavity, for example, due to a remodeling process. The aim of the present study is to identify microbiota surrounding exposed dental implants in patients with and without a history of periodontitis through a deep-sequencing approach.

Methods: An experimental abutment with the same surface and structure as a commercially available dental implant was used. Bacterial DNA was isolated, and the 16S ribosomal RNA gene was amplified and sequenced. Multiplexed tag-encoded sequencing of DNA from the samples was performed, and the reads were processed by metagenomic rapid annotation.

Results: A wide variety of bacteria, 96 species, were identified. The most frequently found bacteria were Fusobacterium nucleatum and Prevotella denticola. Some species generally associated with periodontitis were found to a greater extent in patients without a history of periodontitis. Some bacteria that have never been described as part of the oral microbiome were identified in the present sample.

Conclusions: Analysis of data suggests that the bacteria surrounding exposed dental implants form a diverse microbiome regardless of the periodontal profile of patients. Further research is needed to clarify the role of these microorganisms in the oral environment. J Periodontol 2017;88:1090-1104.

\section{KEY WORDS}

Bacteria; biofilms; dental abutments; dental implants; microbiology; microbiota.

* Oral Surgery and Implantology, Faculty of Medicine and Health Sciences, University of Barcelona, Barcelona, Spain.

† Bellvitge Biomedical Research Institute, Barcelona, Spain.

$\ddagger$ Unit of Genomics, Scientific and Technological Centers, University of Barcelona.

$\S$ Biotechvana, Valencia, Spain. $\longrightarrow$ sseointegrated dental implants have become an important alternative for replacing missing teeth. Despite high survival and success rates of dental implants, biologic complications, mainly peri-implant mucositis and peri-implantitis, are a growing concern. It is estimated that $12 \%$ to $22 \%$ of patients with implants will be diagnosed with peri-implantitis within a short-term follow-up (5 years). ${ }^{1}$

Peri-implantitis is considered an infectious chronic disease that starts with the formation of a heterogeneous biofilm community. ${ }^{2}$ Recent findings suggest a model of pathogenesis in which periodontitis is initiated by a broadly based, dysbiotic, synergistic microbiota, ${ }^{3}$ as opposed to the traditional view of a conventional infectious disease caused by one or more periopathogens such as the "red complex."4 This could also be the case with peri-implant diseases. ${ }^{5}$ The microbiota colonizing implants is still poorly known, and its differences from biofilms around teeth need further investigation. In fact, due to the macrostructure and surface characteristics of implants, biofilm content can be quite different and can favor the presence of pathologic bacteria even in absence of peri-implant diseases. To the authors' knowledge, no data have been published on microbiota formed in patients whose implants have become exposed to the oral cavity due to soft tissue recession but who have no inflammation. 
This knowledge is essential to prevent initiation and progression of peri-implant diseases.

A number of methods have been used to study the microbiota surrounding dental implants. ${ }^{6-8}$ Samples have been collected by rubbing or scratching implants with sterile paper points, curets, or periodontal probes. These procedures recover some of the bacteria but may fail to identify microorganisms that remain firmly attached to the implant surface. Recovering the entire biofilm on exposed implants would be of great value, but this is only possible when the implant is retrieved (i.e., in advanced cases that can only be treated by explantation). A removable abutment mimicking the macrostructure and microstructure of an implant would make it possible to recover an intact biofilm similar to that covering an exposed implant.

Another very important limitation of most studies on this issue is that their techniques, such as oligonucleotide probes, polymerase chain reaction (PCR), and checkerboard DNA-DNA hybridization, do not allow massive bacterial sequencing. ${ }^{6,9-11}$ Pyrosequencing provides a more complete view of the oral microbiome. Using metagenomic techniques and next-generation sequencing technology, the total DNA pool in complex microbiologic samples can be analyzed. This method can detect most species and identify bacteria that cannot be cultivated by standard techniques.

The hypothesis that microbiota colonizing the surface of experimental abutments mimicking exposed dental implants without peri-implant diseases contain a large number of bacterial species, with predominance of Gram-negative bacteria and with differences among patients with and without a previous history of periodontitis, is presented. Therefore, the present study identifies the microbiome formed on abutments that simulate exposed dental implants using pyrosequencing and compares the bacteria of healthy patients and patients with a history of periodontitis.

\section{MATERIALS AND METHODS}

\section{Patient Recruitment}

The experimental non-randomized study consisted of 10 individuals (six males and four females, aged 45 to 84 years; mean age: 60.7 years) with at least one healthy osseointegrated dental implant with a hexagonal external connection, of whom five had a history of periodontitis (periodontitis group, PG), and five were periodontally healthy (healthy group, HG).

Patients were defined as periodontally healthy when they had no attachment loss (AL), no bone loss (BL) measured in periapical radiographs, probing depth (PD) of $<4 \mathrm{~mm}$, and no bleeding on probing (BOP) for at least $70 \%$ of sites. They were considered to have history of periodontitis when they presented $\mathrm{AL} \geq 1 \mathrm{~mm}$ at $>30 \%$ of sites and evidence of BL. It should be pointed out that all patients in PG had been treated, and the disease was under control when they were enrolled in the present trial. All patients met the following inclusion criteria: 1) aged 18 to 90 years; 2) American Society of Anesthesiologists (ASA) health status score ${ }^{12} \leq 3$; 3) osseointegrated implants with hexagonal external connection; 4) not having received final prosthesis; 5) gingival height of $2 \mathrm{~mm}$ from implant shoulder to gingival margin; and 6) sufficient intellectual capacity to understand the study. Study protocol was approved by the Institutional Review Board (Clinical Research Ethics Committee; protocol number 05/14), Dental Hospital, University of Barcelona, Barcelona, Spain, and complied with the Helsinki Declaration of 1975 as revised in 2013. All patients gave written informed consent to participate in the study.

Patients were excluded in the following situations: 1) generalized gingivitis with BOP at $>30 \%$ of sites; 2 ) uncontrolled periodontal disease (PD $\geq 5 \mathrm{~mm}$ with bleeding and/or suppuration); 3) peri-implant disease (implants with bleeding and/or suppuration, and at least one site with radiographic evidence of $\mathrm{BL} \geq 2 \mathrm{~mm}$ ); 4) any periodontal treatment in the 30 days prior to enrollment; or 5) use of antibiotic or antiseptic mouthrinse (bisbiguanides, quaternary ammonium salts, and essential oils) in the 30 days prior to enrollment.

\section{Data Sampling}

A single researcher (BC-A) recruited the patients from February to September 2014 at the Dental Hospital of the University of Barcelona, Hospitalet de Llobregat (Barcelona, Spain) and examined all clinical records. Data retrieved were age, sex, patient health status based on the ASA Physical Status Classification System, ${ }^{12}$ systemic pathologies, current medication, smoking habit (number of cigarettes per day), periodontal disease (periodontal chart with recessions, PD, BOP, and suppuration), and the following implant variables: 1) date of implant placement; 2) diameter; 3) length; 4) position; 5) distance from any nearby implants; 6) width of keratinized mucosa; 7) type of prosthesis; 8) Mombelli peri-implant plaque index (PPI); ${ }^{13}$ 9) Mombelli peri-implant sulcus bleeding in$\operatorname{dex}(\mathrm{PSBI}) ;^{13}$ 10) suppuration; 11) peri-implant PD; and 12) BL. BL was measured on digital periapical radiographs using image processing software.|14

\section{Sample Collection and DNA Isolation}

The abutments used, specifically fabricated for this purpose, were designed to have the same macroscopic and microscopic shape as an implant with a bioabsorbable blast media surfacell (see supplementary Fig. 1 in online Journal of Periodontology).

After a thorough explanation of the study objectives, a healing abutment was replaced by the experimental

\| ImageJ software, US National Institutes of Health, Bethesda, MD. If Mozo-Grau dental implants, Mozo-Grau, Valladolid, Spain. 
abutment (see supplementary Fig. 1 in online Journal of Periodontology). Patients were instructed to refrain from cleaning the abutment and using toothpaste or mouthrinse solutions during the study period.

After 14 days, the experimental abutment was carefully removed, placed in a sterile snap-cap tube, and transported to the laboratory in $<1$ hour in a portable refrigerator at $4^{\circ} \mathrm{C}$. The abutment was screwed to an implant analog placed inside the tube, allowing the biofilm to remain intact without touching the tube wall.

All patients were enrolled in a peri-implant maintenance program, and a final prosthesis was made.

After the abutment was received at the laboratory, it was unscrewed, stored in a $1.5-\mathrm{mL}$ microcentrifuge tube, and frozen at $-80^{\circ} \mathrm{C}$ until further analysis. The abutment was rinsed with phosphate-buffered saline and vortexed for 5 minutes to release the bacteria. Total DNA was purified with a DNA purification kit, ${ }^{\#}$ according to the manufacturer's protocol for buccal swabs. The amount of DNA extracted was calculated using a scientific instrumentation system. ${ }^{* *}$

\section{Statistical Analyses}

Variable regions V1, V2, V3, V4, and V5 of the $16 \mathrm{~S}$ ribosomal RNA (rRNA) gene were amplified with a multiplex PCR system ${ }^{\dagger \dagger}$ and sequenced with a titanium sequencing kit. ${ }^{\ddagger}$

PCRs for V1-V3 and V5-V3 primers were set up with annealing temperatures of $56^{\circ} \mathrm{C}$ and $50^{\circ} \mathrm{C}$, respectively. Two replicate PCRs were performed and pooled for each sample. Amplicon library was cleaned with a PCR purification system, $\$ \S$ according to the manufacturer's instructions. Amplicon concentration was estimated using an assay kit.|l|

Multiplexed tag-encoded sequencing of DNA from the samples was performed on a pyrosequencing platform. It

Primers used to amplify the 16S rRNA gene and to introduce multiplex identifiers to identify amplicons or samples are available on the National Institutes of Health Human Microbiome Project website. ${ }^{15}$

The resulting fast files were preprocessed with a quality control and data preprocessing tool ${ }^{16}$ by size (more than $50 \mathrm{bp}$ ), quality (minimum quality 30 ), and $N$ content (rejecting reads with $>5 \%$ of $\mathrm{Ns}$ and removing terminal Ns).

The reads were processed through metagenomic rapid annotation using subsystems technology (MGRAST $)^{17}$ based on hierarchical classification with the Ribosomal Database Project (RDP; release 11). MGRAST default clustering parameters within the basic local alignment search tool-like alignment tool algorithm were used.

Each read was taxonomically assigned down to the genus and species level with $80 \%$ confidence threshold. Reads giving no bacterial hits were excluded. Artificial replicate sequences produced by sequencing artifacts were removed. ${ }^{18}$

To estimate bacterial diversity, the number of operational taxonomic units (OTUs) in the samples was determined, and rarefaction analysis was performed. Rarefaction curves were obtained by plotting the number of observed OTUs against the number of sequences, using the MG-RAST platform ${ }^{17}$ and the RDP database. ${ }^{19,20}$

To estimate total diversity, sequences were clustered at a standard threshold of $98 \%$ nucleotide identity over a $90 \%$ sequence alignment length to allow minimal flexibility and to minimize false positives. Rarefaction curves were obtained using the RDP pyrosequencing pipeline (Fig. 1A). Venn analysis (Fig. 1B) and principal component analysis (PCA) were performed, and heatmaps were generated using a statistical package. ${ }^{21}$ Venn analysis was run on taxonomic diversity data. PCA analysis was run on taxonomic diversity and abundance of each individual sample and on the average of each group (i.e., PG and HG) (Fig. 1C).

Microbial communities were compared by statistical analysis using a distance metric matrix. ${ }^{22}$ This analysis compares the $16 \mathrm{~S}$ estimated diversity through a phylogenetic approach that takes taxonomically assigned and unassigned reads into account (Fig. 2). Heatmap analysis of taxonomic diversity and abundance was done for each sample (Fig. 3).

\section{RESULTS}

After extracting metagenomic data, quality of the readings was assessed by a quality control tool. ${ }^{\# \#}$ Sequencing samples were of excellent quality (Phred values $>28$ ). Unknown reads (not identified as rRNA genes) varied among samples, ranging from $0.08 \%$ to $0.31 \%$. Samples used in this study were deposited in the GenBank under accession numbers SAMN06116059 to SAMN06116068.

\section{Oral Microbial Community}

The number of reads (filtered and assigned) and number of taxonomic assignations (genus and species) are listed in Table 1. Although microbiota-based rarefaction curves (Fig. 1A) failed to reach saturation phase, the slope of the curves become notably less pronounced.

\# QiAamp DNA minikit, Qiagen, Hilden, Germany.

** Qubit system, Thermo Fisher Scientific, Waltham, MA.

$\dagger \dagger$ FastStart High Fidelity PCR Systems, Roche, Mannheim, Germany.

¥ GS Junior titanium sequencing kit, Roche.

$\S \S$ Agencourt AMPure beads, Beckman Coulter, Brea, CA

Qubit dsDNA HS assay kit, Thermo Fisher Scientific.

II GS Junior platform, Roche Applied Science, Indianapolis, IN.

\#\# FastQC pipelines, Babraham Bioinformatics, Babraham Institute, Cambridge, U.K. 
A

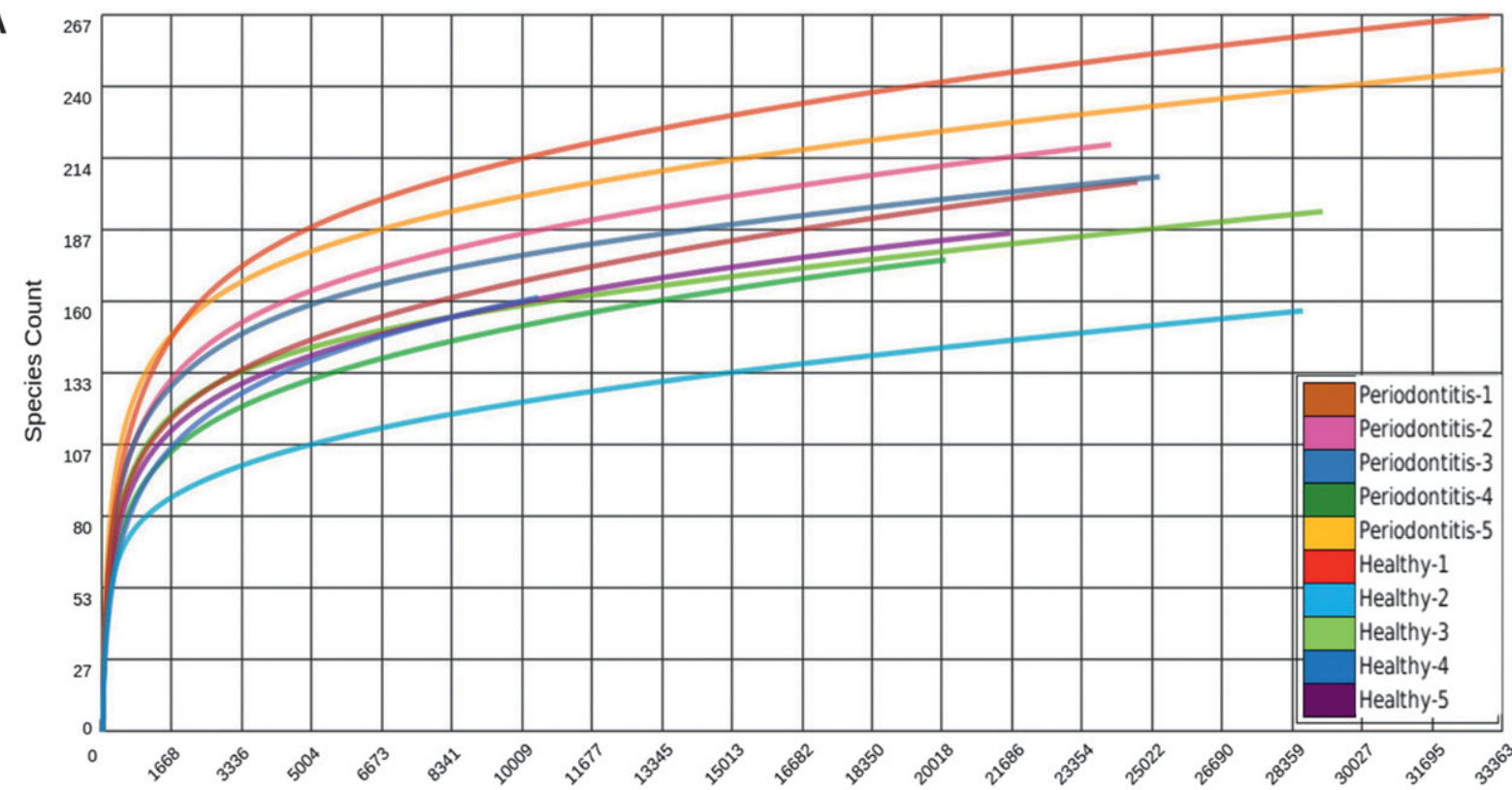

Number of Sequences Sampled

B

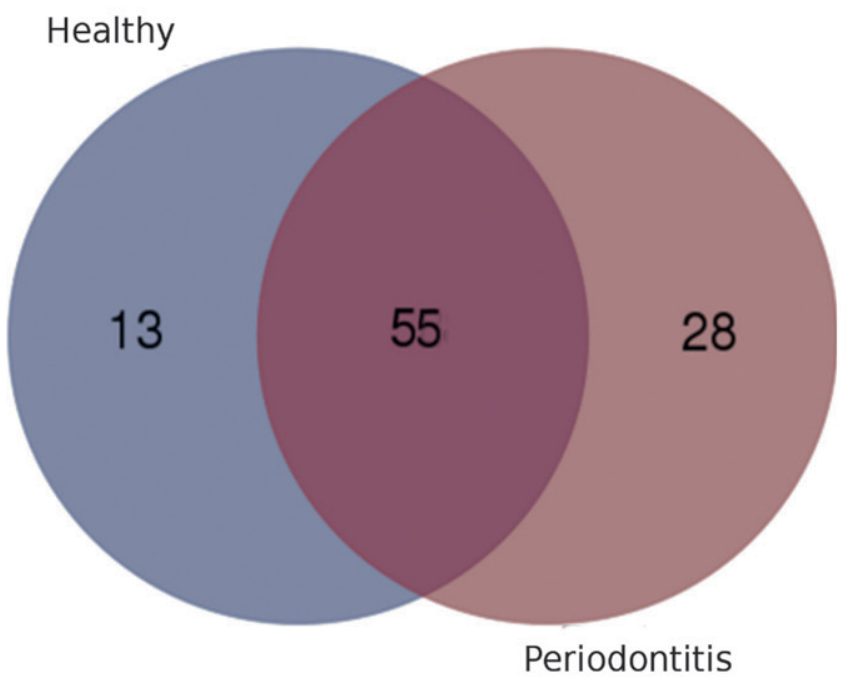

C

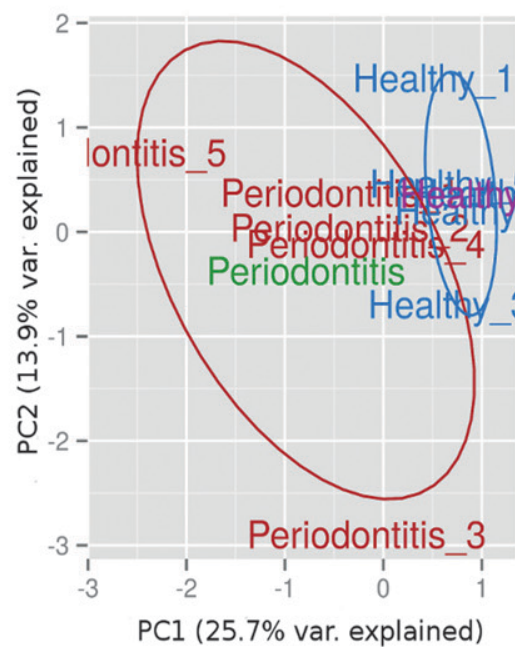

a Periodontitis

a Periodontitis_average

a Healthy

at Healthy_average

Figure I.

A) Rarefaction plot showing a curve of annotated species richness. This curve represents the total number of distinct species annotations as a function of the number of sequences sampled. B) Venn diagram demonstrating bacterial taxonomic distribution between diseased and healthy samples. C) PCA relationships among groups of samples. Circles include samples belonging to the same group.

Taxonomic results showed 96 different species: 55 common to both groups, 28 only found in PG, and 13 specific to HG (Figs. 1B and 4; Table 2). Both groups showed homogeneous distribution (clearer in HG). The heatmap (Fig. 3) clearly shows a number of species with greater representation in the overall number of bacteria, overexpressed particularly in HG.

The most abundant bacterial species among those $>5 \%$ of the total were as follows: 1) PG: Fusobacterium nucleatum, Prevotella oris, Prevotella denticola, and Prevotella melaninogenica; 2) HG: F. nucleatum, $P$. melaninogenica, Rothia dentocariosa, P. oris, and Veillonella parvula; and 3) overall: F. nucleatum, P. oris, $P$. melaninogenica, $R$. dentocariosa, and $P$. denticola. The most commonly found was $F$. nucleatum, overrepresented in HG. The genus Prevotella was the most common overall, and $P$. denticola was overrepresented in PG, with 13 times higher abundance than in HG. 


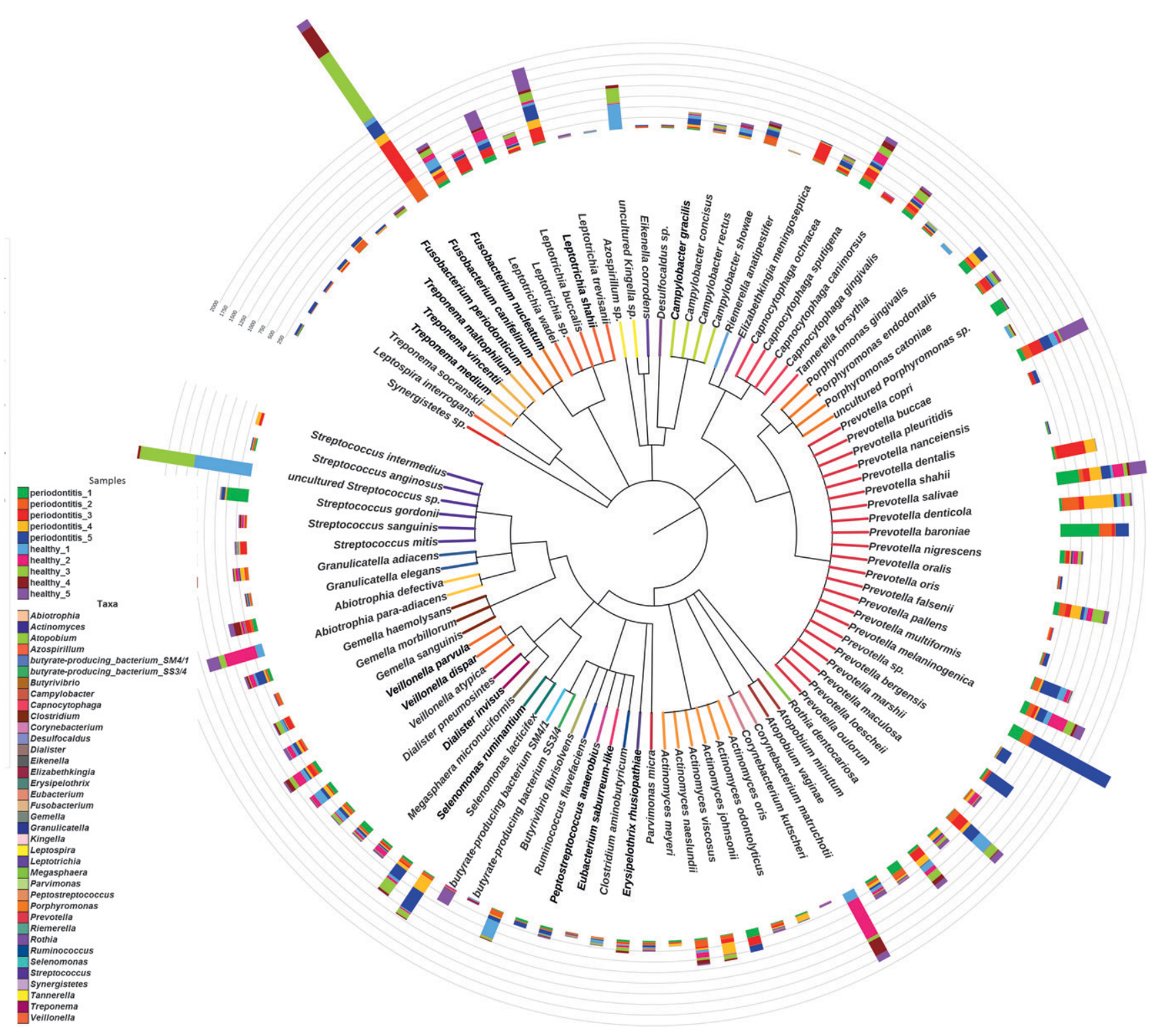

\section{Figure 2.}

Bacteriome cladogram with pyrosequencing datasets of the two groups of samples (PG and HG: pool I and pool 2). The RDP database ${ }^{20}$ was used as the annotation source, and a minimum identity cutoff of $98 \%$ was applied. Colors for the genus branches are indicated in the taxa section of the key. Bars in the external circle indicate abundance of the term in each sample. Colors of the samples are indicated in the samples section of the key.

Sixteen of the bacteria found have been related to microbial complexes of subgingival plaque ${ }^{4}$ (see supplementary Table 1 in online Journal of Periodontology).

Bacteria from the green and purple clusters seemed to be more numerous than others, except for the above-mentioned bacteria and Campylobacter showae (orange cluster).

Regarding differences between groups (Table 2), periodontopathogens were not more prevalent in PG than in HG. Moreover, Porphyromonas gingivalis was only found in healthy individuals. In contrast, first colonizers such as Streptococcus anginosus and Streptococcus intermedius were only found in PG.
Of the 96 bacteria identified, 19 were not in the Human Oral Microbiome Database (HOMD) ${ }^{23}$ or the CORE Microbiome Database. ${ }^{24}$

\section{DISCUSSION}

Implant surfaces are designed to enhance osseointegration. When bone remodeling or loss occurs and areas of the implant surface become exposed to the oral environment, saliva biopolymers form a film that becomes the interface between the implant surface and the first microorganisms. Many characteristics of the titanium implant surface, such as roughness, hydrophobicity, and charge, affect bacterial adhesion. ${ }^{25}$ 

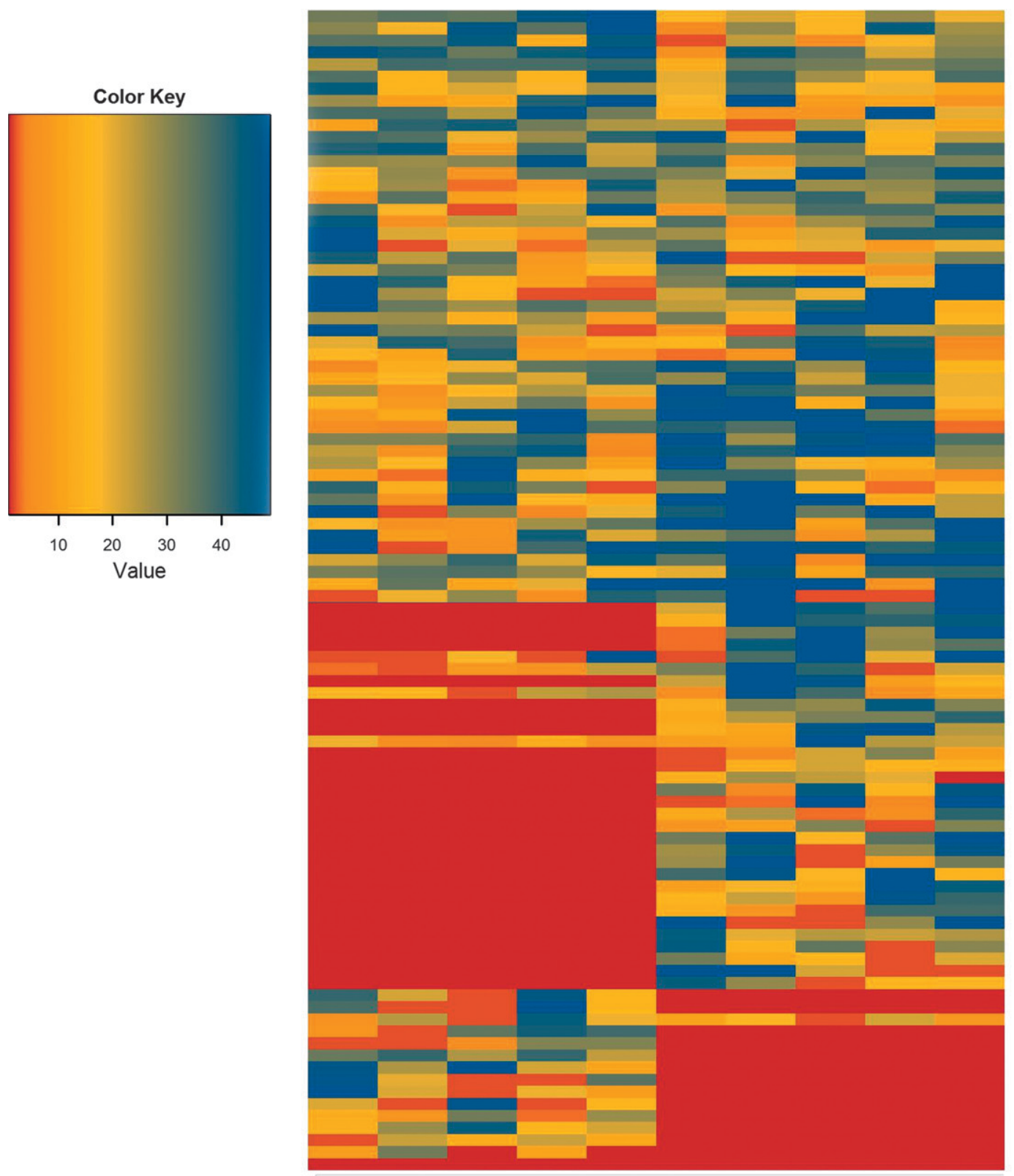

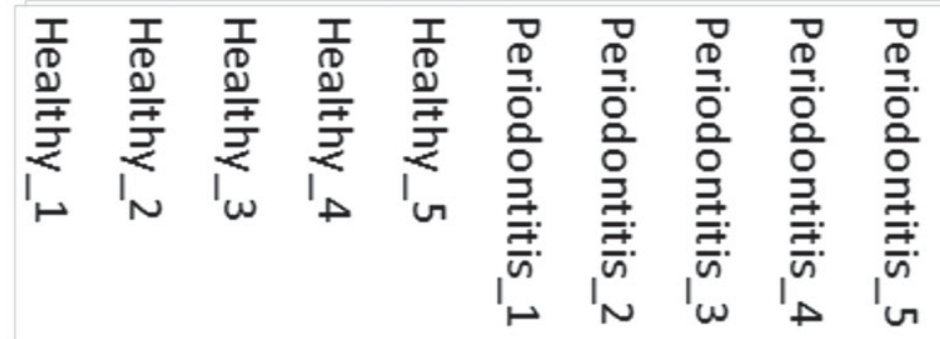

Clostridium aminobutyricum Granulicatella adiacens

Prevotella ${ }^{-}$oulorum

Tannerella_forsythia Campylobăcter_concisus

eptotrichia sp

Streptococcus_mitis

Campylobacter_showae

Campylobacter_gracilis

Gemelia haemōlysans

Leptotrichia_wadei

Megasphaera_micronuciformis

Cranulicatella_elegans

Actinomyces odontolyticus

Atopobium - minutum

Srevotecoccus sanguinis

Leptotrichĩa buccalis

Actinomyces viscosus

Prevotella_salivae

Erysipelothrix raburreum

Fusobacterium canifelinum

Abiotrophia_défectiva.

Serphyromonas catomian

Parvimonas micra

Capnocytophaga_sputigena

Prevotella_denticola

Selenomoñas ruminantium

Atopobium vāginae

Prevotella- multiformis

Veillonella àtypica

Dialister pneumosintes

Prevotella_maculosa

Veillonella parvula

Capnocytophaga_ gingivalis

Fusobacterium_nucleatum

revotella_shahii

Prevotella_marshii

Prevotella_baronia

Capnocytophaga canimorsus

Streptococcus goordonii

Treponema_vincentii

Peptostreptococcus_anaerobius

Streptococcus anginosus

Gemella morbillorum

Rothia dentocariosa

Desulfocaldus sp,

uncultured Porphyromonas_s $p$,

Ruminococcus_flavefaciens

Prevotella pleuritidis

Actinomycos oris

Leptospira_interrogans

Treponema_medium

Porphyromonas_endodontalis Elizabethkingia_meningoseptic

Streptococcus_intermedius

Streptococcus
Prevotella_sp,

Actinomyces_meyeri
Actinomyces_johnsoni

Synergistetes_sp,

Prevotelläcopri

Porphyromonas gingivalis

Gemella_sanguinis

Corynebacterium kutscheri

uncultured Kingella $s p$,

Corynebacterium_matruchotii

Azospirillum sp
Riemerella_anatipestifer
butyrate-producing bacteri

butyrate-producing_bacterium_SM4/1

butyrate-producing_bacterium_sS3/4

butyrate-producing_bacterium
uncultured_Streptococcus_sp,
Prevotella_nanceiensis

Figure 3.

Heatmap visualization of changes in bacterial diversity among groups (HG and PG, represented by individual samples). 


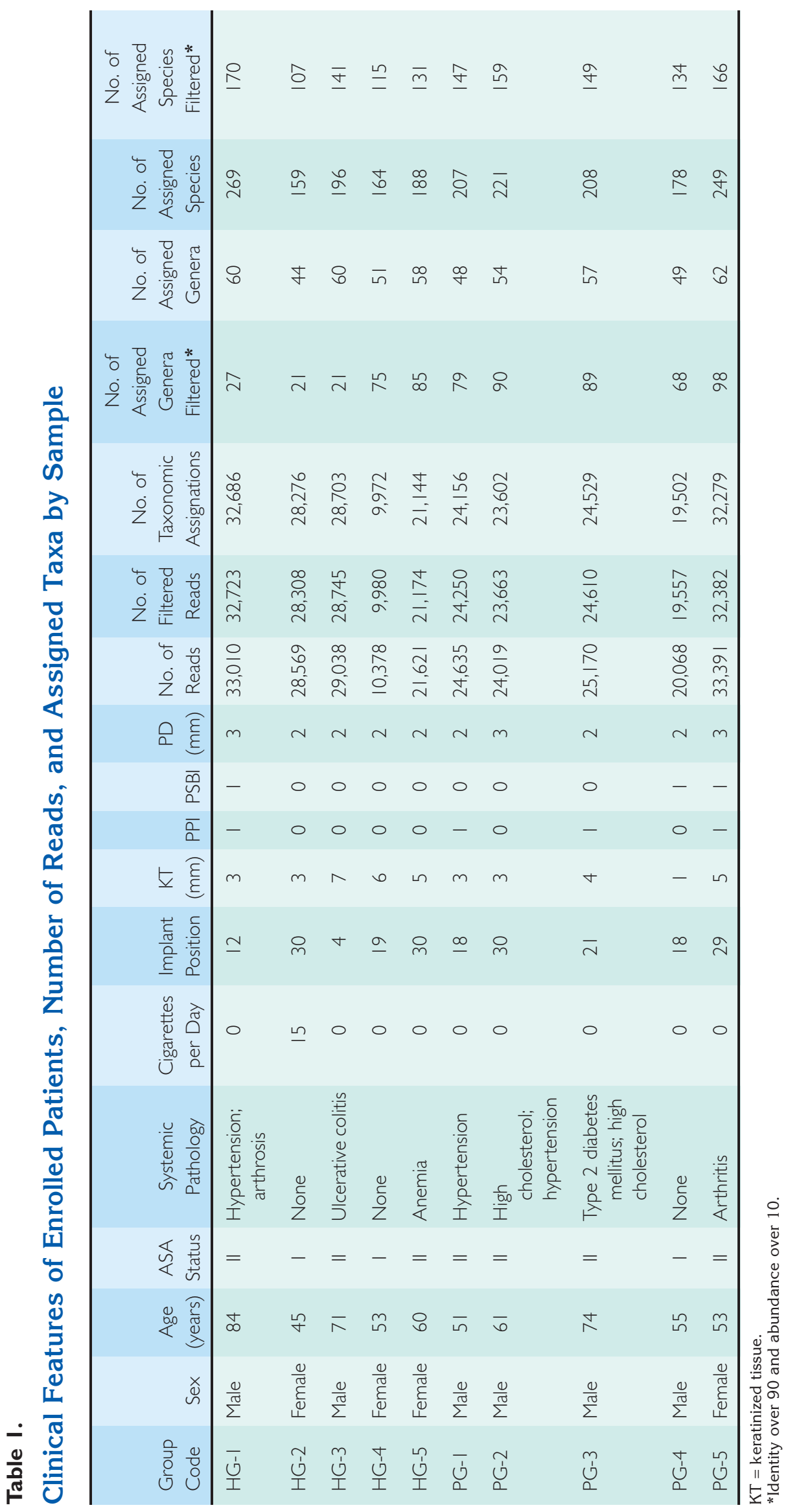



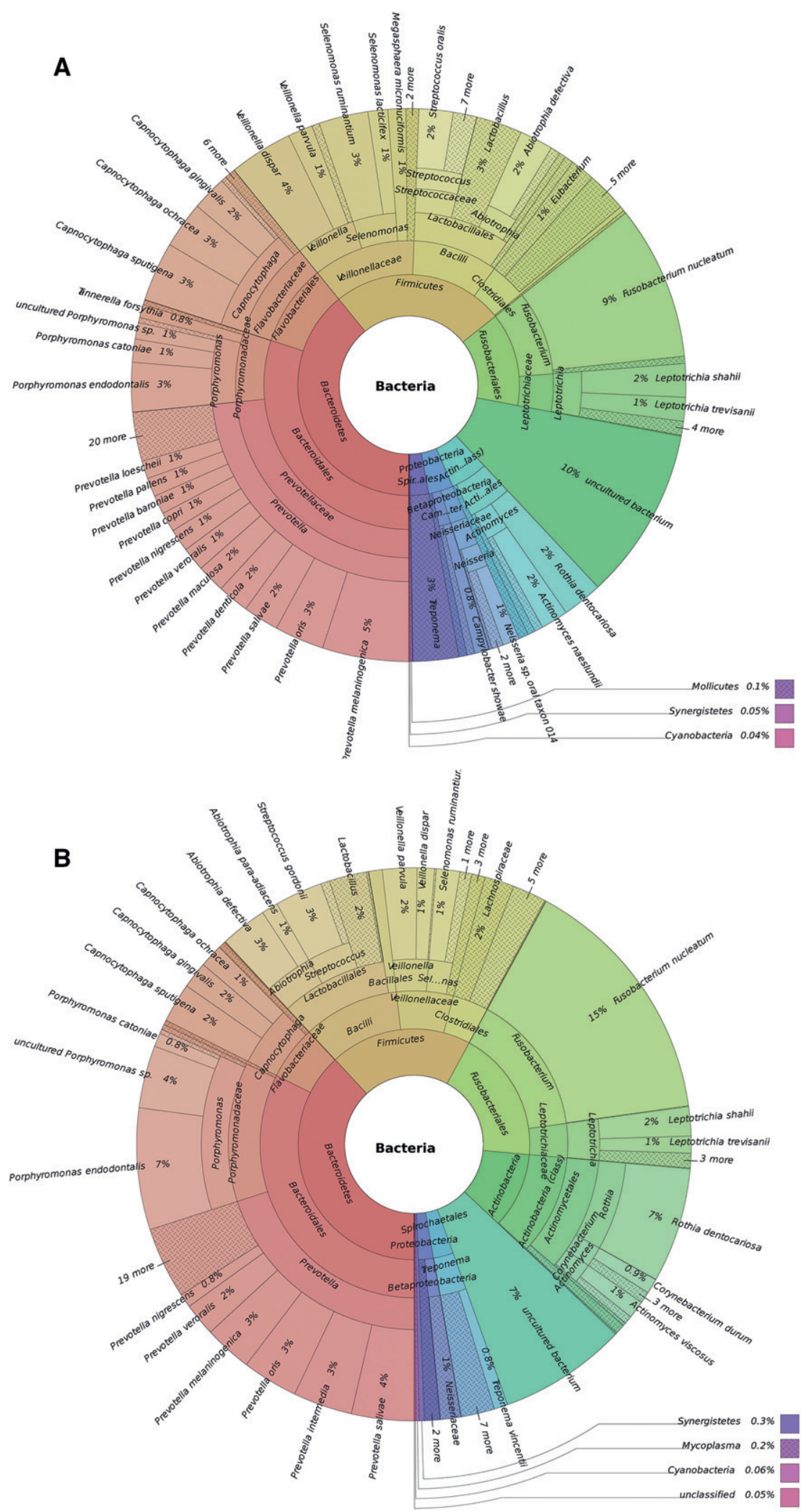

\section{Figure 4.}

Taxonomic spectrum visualized with Krona chart of counts in the metagenome. Circles represent taxonomic classifications in ascending order up to the family level (outermost circle). Less abundant taxa are listed outside the charts together with their relative abundance. Data are obtained from raw data using the MG-RAST server. A) PG samples pool. B) HG samples pool. 


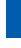

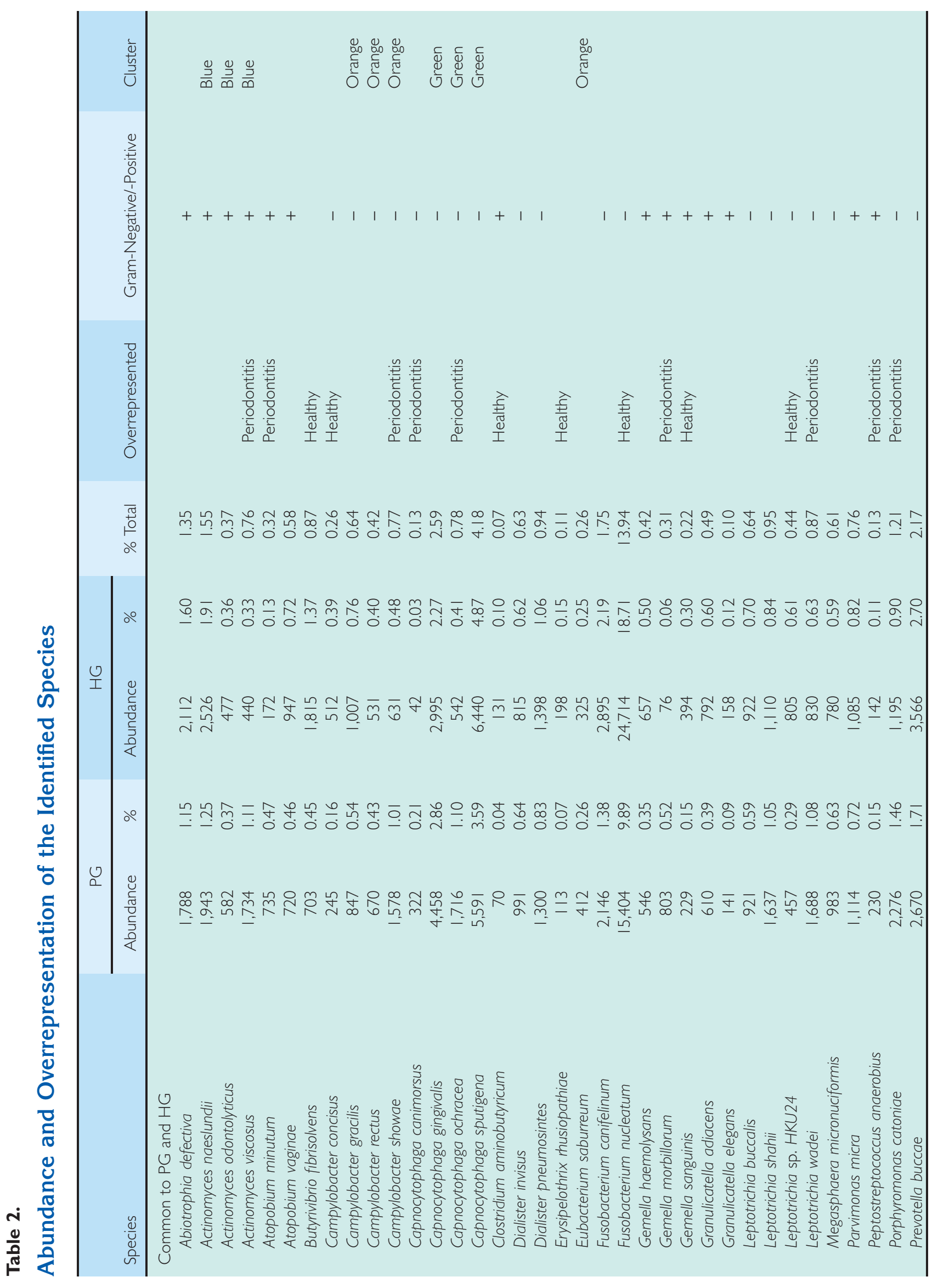




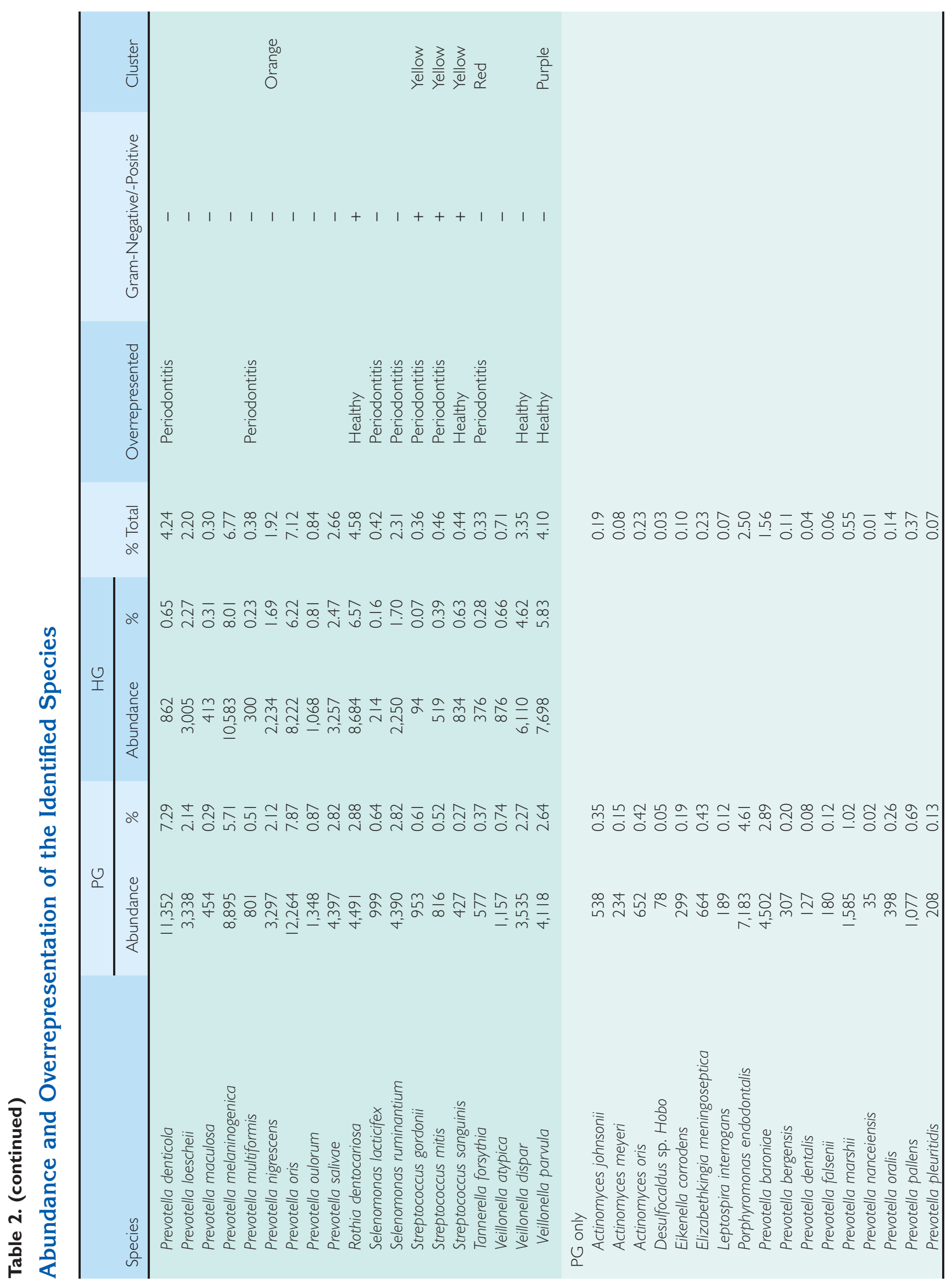




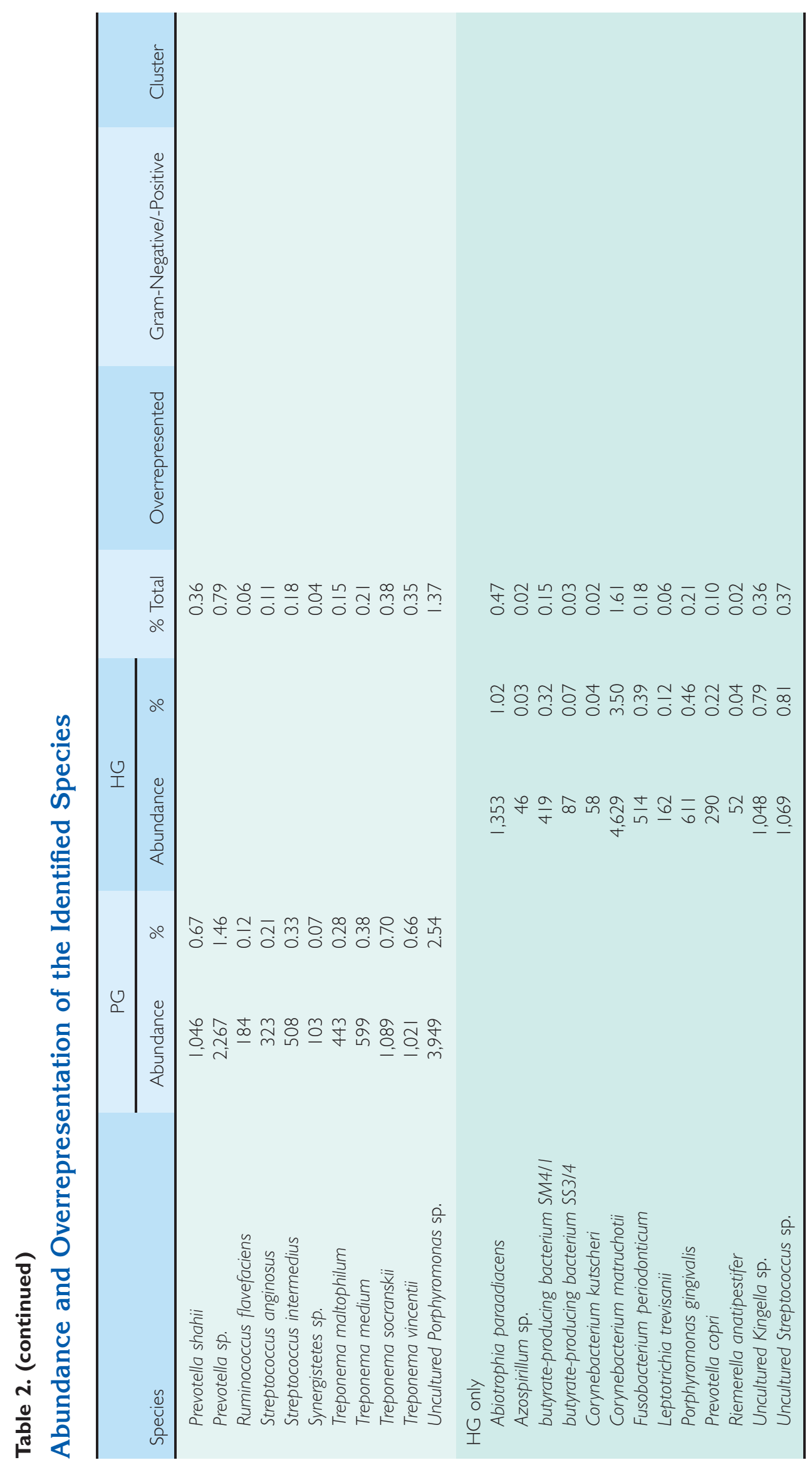


The present study adds new information regarding microbiota formed on implants when they become exposed to the oral cavity without associated inflammation (i.e., in soft tissue recession or bone remodeling processes). These data can also be useful when peri-implantitis patients are surgically treated with a resective approach. In these cases, after surface decontamination, an apically repositioned flap is made to reduce peri-implant pockets, thus exposing the rough surface of the implant.

One of the main limitations of this study is the reduced sample size, which may jeopardize generalization of outcomes. Also, the fact that all samples were collected after 14 days did not allow a study of the evolution of biofilm formation.

Periodontal disease is a known risk factor for periimplantitis and one of the explanations, apart from patient susceptibility, is that periodontally involved teeth may act as a reservoir for periodontal pathogens that can colonize the implant surface. ${ }^{26}$ However, a study using an open-ended molecular approach showed that in $85 \%$ of participants, $<8 \%$ of species were shared between teeth and implants, suggesting that microbiology of peri-implantitis and periodontitis might be quite different. ${ }^{27}$ Data from the present study, although obtained from healthy sites, seem to support these results, as species generally associated with diseased implants, such as $P$. gingivalis, are detected in healthy implants but only in periodontally healthy individuals. $P$. gingivalis might have an important role in peri-implant diseases as it has been described as an "enhancer species" that is involved in coaggregation stages during biofilm maturation. ${ }^{28}$

On the other hand, many identified bacteria such as Streptococcus sanguinis, Actinomyces naeslundii, Campylobacter rectus, Parvimonas micra, and Granulicatella adiacens or the genera Fusobacterium, Actinomyces, Veillonella, Atopobium, Gemella, Rothia, and Leptotrichia have been associated with healthy implants in previous studies. ${ }^{8,28-30}$ Special consideration should be taken with the genera Actinomyces and Veillonella as most authors ${ }^{28,31}$ agreed to finding them more frequently in healthy implants, and none of the revised studies associate them with peri-implantitis. ${ }^{8,28-33}$ Presence of these bacteria, together with Streptococcus mitis and S. sanguinis, may play a protective role regarding peri-implant diseases. ${ }^{34,35}$

Prevotella spp. were found widely in both PG and HG, and $P$. denticola was one of the most abundant bacteria. It has been associated with periodontal disease as strongly as the classic red complex, ${ }^{36}$ but it should be remembered that patients in PG had PD $<4 \mathrm{~mm}$ and at least $70 \%$ of sites with no BOP. Therefore, presence of this genus in both groups may suggest that it is only pathogenic when the bacterial balance is disturbed or when there is host susceptibility.

Another most abundant bacterium was $F$. nucleatum. It is known to mediate between the first and subsequent colonizers and interact with host cells, facilitating coaggregation with periodontopathogens such as $P$. gingivalis. ${ }^{37}$ Some authors suggest that $F$. nucleatum infection facilitates attachment of $P$. gingivalis to the gingival fibroblast, and consequently these two bacteria are often found together. ${ }^{38}$ Interestingly, $F$. nucleatum was abundant in both groups (15,404 in PG and 24,714 in HG), whereas P. gingivalis was not identified in PG but had an abundance of 611 in HG.

Previous publications concluded that in periodontally healthy individuals, $P$. gingivalis and $A g$ gregatibacter actinomycemcomitans were never ${ }^{39}$ or rarely ${ }^{40}$ found on implants. The present study contradicts this statement, as $P$. gingivalis was specifically found in HG. This disparity might be explained by the different analytic method (pyrosequencing), which in the authors' opinion affords more complete and detailed data gathering and should be implemented more frequently in the future.

The microbiome surrounding teeth has been shown to be significantly more diverse than that around implants. ${ }^{41,42}$ In addition, the rate of traditional pathogens around implants has been reported to be lower than that around teeth in both healthy and diseased patients. ${ }^{43}$ Cortelli et al. ${ }^{43}$ also pointed out that early colonizers of rough implant surfaces might constitute a different bacterial microbiome from that of periodontal diseases. The present report shows that individuals have an important variability regarding the composition of biofilm. This indicates that studies with large samples are required. It would be interesting to analyze whether this variability is related with the different progression patterns of BL found in peri-implantitis.

Streptococcus, Granulicatella, and Gemella were present in both PG and HG, in agreement with a previous study. ${ }^{44}$ These bacteria are considered symbionts, with a high proportion returning to pockets after periodontal treatments. ${ }^{45}$

Regarding differences among groups, special mention should be made of four bacteria with $>1 \%$ abundance: Porphyromonas endodontalis, Prevotella baroniae and an uncultured Porphyromonas sp. in PG and Corynebacterium matruchotii in HG. P. endodontalis is found in symptomatic oral infections, such as endodontic infections and periodontal pockets, but also in asymptomatic cases. It shows low virulence in experimental monoinfections but seems to play an important role in anaerobic mixed infections. ${ }^{46} P$. baroniae has been described as a causal agent of endodontic abscesses. ${ }^{47} \mathrm{C}$. matruchotii is considered part of normal oral microbiota. ${ }^{48}$ 
High prevalence of Gram-negative bacteria in HG and Gram-positive bacteria in PG is surprising, as earlier studies demonstrated Gram-negative prevalence in oral microbioma. ${ }^{2}$

Although 19 bacteria were not registered in the HOMD or CORE databases, five had been reported previously, including Prevotella bergensis, Leptotrichia sp. HKU24, and Prevotella copri. The remaining 14 have been identified in human infections in other areas of the body or found in insects, contaminated water, dogs, cats, or birds (see supplementary Table 1 in online Journal of Periodontology). Contamination of samples during transport was highly unlikely because the snap-tubes were sterile, and the abutment did not touch its walls (it was firmly screwed to a sterile implant replica). Future research should examine whether these microorganisms play an important role in peri-implant diseases. This is quite an important finding, and once again indicates the importance of using metagenomic analysis techniques. Other microbiologic methods such as checkerboard DNA-DNA hybridization are indeed extremely accurate and have high sensitivity (>92.5\%) and specificity $(100 \%)$, but are clearly insufficient to detect composition of the microbiome surrounding implants. ${ }^{49}$ Likewise, the sample collection method might cause important discrepancies among studies. Biofilms collected with curets can result in lower bacterial counts due to implant topography, ${ }^{11}$ and sterile paper points can be a source of foreign bacteria. ${ }^{50}$ This is an important advantage of the present method to recover biofilm. Other authors have previously reported use of abutments with different roughnesses ${ }^{51}$ for this purpose, but with no threads. Thus, a study comparing different biofilm sampling methods would be of interest.

\section{CONCLUSIONS}

A wide variety of bacteria (96 species) were found around abutments simulating exposed dental implants without inflammation in 10 individuals. The most frequently found bacteria were $F$. nucleatum and $P$. denticola. Some species generally associated with periodontitis were more commonly found in patients without history of periodontitis.

A large number of bacteria that had never been described as part of the oral microbiome were found in the present sample. Further research with larger samples is needed to clarify the role of these microorganisms in the oral environment.

\section{ACKNOWLEDGMENTS}

The authors thank Dr. Rubén León and Dr. Vanessa Blanc from the Research and Development and Microbiology Departments of Dentaid SL (Cerdanyola del Vallés, Spain) for critical review of the manuscript. The authors also thank Mary Georgina Hardinge (native British freelance translator, Valencia, Spain) for
English language editing of the manuscript. Dr. Figueiredo reports grants from the Faculty of Dentistry, University of Barcelona (Spain) and non-financial support from Mozo-Grau (Valladolid, Spain) during conduct of the study. Also, he reports grants, personal fees, and non-financial support from Mozo-Grau (Valladolid, Spain) and personal fees from BioHorizons Ibérica (Madrid, Spain), Inibsa Dental (Lliça de Vall, Spain), DENTSPLY implants Iberia (Barcelona, Spain), and ADIN implants (Afula, Israel) outside the submitted work. In addition, Dr. Figueiredo has a patent "Biofilm collector abutment" pending to Mozo-Grau, Rui Figueiredo, and Eduard Valmaseda-Castellón. Dr. Valmaseda-Castellón reports grants from the Faculty of Dentistry, University of Barcelona (Spain) and non-financial support from Mozo-Grau (Valladolid, Spain) during conduct of the study. Also, he reports grants, personal fees, and non-financial support from Mozo-Grau (Valladolid, Spain) and personal fees from BioHorizons Ibérica (Madrid, Spain), Inibsa Dental (Lliça de Vall, Spain), and DENTSPLY implants Iberia (Barcelona, Spain) outside the submitted work. In addition, Dr. Valmaseda-Castellón has a patent "Biofilm collector abutment" pending to Mozo-Grau, Rui Figueiredo, and Eduard Valmaseda-Castellón. Dr. Cortés-Acha reports grants from the Faculty of Dentistry, University of Barcelona (Spain) and non-financial support from Mozo-Grau (Valladolid, Spain) during conduct of the study. The present research was conducted by the Dental and Maxillofacial Pathology and Therapeutics research group at the IDIBELL Institute (L'Hospitalet de Llobregat, Spain) and funded by a postgraduate research grant from the Faculty of Dentistry, University of Barcelona (€4.560).

\section{REFERENCES}

1. Mir-Mari J, Mir-Orfila P, Figueiredo R, Valmaseda-Castellón E, Gay-Escoda C. Prevalence of peri-implant diseases. A cross-sectional study based on a private practice environment. J Clin Periodontol 2012;39:490-494.

2. Quirynen M, De Soete M, van Steenberghe D. Infectious risks for oral implants: A review of the literature. Clin Oral Implants Res 2002;13:1-19.

3. Hajishengallis G, Lamont RJ. Beyond the red complex and into more complexity: The polymicrobial synergy and dysbiosis (PSD) model of periodontal disease etiology. Mol Oral Microbiol 2012;27:409-419.

4. Socransky SS, Haffajee AD, Cugini MA, Smith C, Kent RL Jr. Microbial complexes in subgingival plaque. $J$ Clin Periodontol 1998;25:134-144.

5. Murray JL, Connell JL, Stacy A, Turner KH, Whiteley M. Mechanisms of synergy in polymicrobial infections. J Microbiol 2014;52:188-199.

6. Shibli JA, Melo L, Ferrari DS, Figueiredo LC, Faveri M, Feres M. Composition of supra- and subgingival biofilm of subjects with healthy and diseased implants. Clin Oral Implants Res 2008;19:975-982.

7. Kumar PS, Mason MR, Brooker MR, O’Brien K. Pyrosequencing reveals unique microbial signatures associated 
with healthy and failing dental implants. J Clin Periodontol 2012;39:425-433.

8. Zheng H, Xu L, Wang Z, et al. Subgingival microbiome in patients with healthy and ailing dental implants. Sci Rep 2015;5:10948.

9. Quirynen M, Vogels R, Peeters W, van Steenberghe D, Naert I, Haffajee A. Dynamics of initial subgingival colonization of "pristine" peri-implant pockets. Clin Oral Implants Res 2006;17:25-37.

10. Renvert S, Roos-Jansåker AM, Lindahl C, Renvert H, Rutger Persson G. Infection at titanium implants with or without a clinical diagnosis of inflammation. Clin Oral Implants Res 2007;18:509-516.

11. Gerber J, Wenaweser D, Heitz-Mayfield L, Lang NP, Persson GR. Comparison of bacterial plaque samples from titanium implant and tooth surfaces by different methods. Clin Oral Implants Res 2006;17:1-7.

12. ASA House of Delegates. American Society of Anesthesiologists - ASA Physical Status Classification System. Available at: http://www.asahq.org/ resources/clinical-information/asa-physical-statusclassification-system. Accessed April 20, 2017.

13. Mombelli A, van Oosten MA, Schurch E Jr., Land NP. The microbiota associated with successful or failing osseointegrated titanium implants. Oral Microbiol Immunol 1987;2:145-151.

14. García-García M, Mir-Mari J, Benic GI, Figueiredo R, Valmaseda-Castellón E. Accuracy of periapical radiography in assessing bone level in implants affected by peri-implantitis: A cross-sectional study. J Clin Periodontol 2016;43:85-91.

15. Jumpstart Consortium Human Microbiome Project Data Generation Working Group. 16S 454 Sequencing Protocol HMP Consortium 2010. Available at: http:// www.hmpdacc.org/doc/16S_Sequencing_SOP_4.2.2. pdf. Accessed January 25, 2016.

16. Schmieder R, Edwards R. Quality control and preprocessing of metagenomic datasets. Bioinformatics 2011; 27:863-864.

17. Meyer F, Paarmann D, D'Souza M, et al. The metagenomics RAST server - A public resource for the automatic phylogenetic and functional analysis of metagenomes. BMC Bioinformatics 2008;9:386.

18. Gomez-Alvarez V, Teal TK, Schmidt TM. Systematic artifacts in metagenomes from complex microbial communities. ISME J 2009;3:1314-1317.

19. Wang Q, Garrity GM, Tiedje JM, Cole JR. Naive Bayesian classifier for rapid assignment of rRNA sequences into the new bacterial taxonomy. Appl Environ Microbiol 2007;73:5261-5267.

20. Cole JR, Wang Q, Cardenas E, et al. The Ribosomal Database Project: Improved alignments and new tools for rRNA analysis. Nucleic Acids Res 2009;37:D141-D145.

21. Futami R, Muñoz-Pomer A, Viu JM, et al. GPRO: The professional tool for management, functional analysis and annotation of omic sequences and databases. Biotechuana Bioinforma 2011;1:1-5.

22. Chen J, Bittinger K, Charlson ES, et al. Associating microbiome composition with environmental covariates using generalized UniFrac distances. Bioinformatics 2012; 28:2106-2113.

23. Chen T, Yu W-H, Izard J, Baranova OV, Lakshmanan A, Dewhirst FE. The Human Oral Microbiome Database: A Web Accessible Resource for Investigating Oral Microbe Taxonomic and Genomic Information. Database (Oxford) 2010;2010:baq013.
24. OSU CORE Database, Oral Microbiome. Available at: http://microbiome.osu.edu/sequences. Accessed November 21, 2016.

25. Teughels W, Van Assche N, Sliepen I, Quirynen M. Effect of material characteristics and/or surface topography on biofilm development. Clin Oral Implants Res 2006;17 (Suppl. 2):68-81.

26. Renvert S, Roos-Jansåker AM, Lindahl C, Renvert H, Rutger Persson G. Infection at titanium implants with or without a clinical diagnosis of inflammation. Clin Oral Implants Res 2007;18:509-516.

27. Dabdoub SM, Tsigarida AA, Kumar PS. Patient-specific analysis of periodontal and peri-implant microbiomes. $J$ Dent Res 2013;92(Suppl. 12):168S-175S.

28. Rickard AH, Gilbert P, High NJ, Kolenbrander PE, Handley PS. Bacterial coaggregation: An integral process in the development of multi-species biofilms. Trends Microbiol 2003;11:94-100.

29. Neilands J, Wickström C, Kinnby B, et al. Bacterial profiles and proteolytic activity in peri-implantitis versus healthy sites. Anaerobe 2015;35(Pt A):28-34.

30. da Silva ES, Feres M, Figueiredo LC, Shibli JA, Ramiro FS, Faveri M. Microbiological diversity of peri-implantitis biofilm by Sanger sequencing. Clin Oral Implants Res 2014;25:1192-1199.

31. Tamura N, Ochi M, Miyakawa H, Nakazawa F. Analysis of bacterial flora associated with peri-implantitis using obligate anaerobic culture technique and 16S rDNA gene sequence. Int J Oral Maxillofac Implants 2013;28: $1521-1529$.

32. Persson GR, Renvert S. Cluster of bacteria associated with peri-implantitis. Clin Implant Dent Relat Res 2014; 16:783-793.

33. Eick S, Ramseier CA, Rothenberger K, Brägger U, Buser D, Salvi GE. Microbiota at teeth and implants in partially edentulous patients. A 10-year retrospective study. Clin Oral Implants Res 2016;27:218-225.

34. Quirynen M, De Soete M, Dierickx K, van Steenberghe $D$. The intra-oral translocation of periodontopathogens jeopardises the outcome of periodontal therapy. A review of the literature. J Clin Periodontol 2001;28: 499-507.

35. Stingu C-S, Eschrich K, Rodloff AC, Schaumann R, Jentsch H. Periodontitis is associated with a loss of colonization by Streptococcus sanguinis. J Med Microbiol 2008;57:495-499.

36. Vartoukian SR, Palmer RM, Wade WG. Diversity and morphology of members of the phylum "synergistetes" in periodontal health and disease. Appl Environ Microbiol 2009;75:3777-3786.

37. Ang MY, Dutta A, Wee WY, Dymock D, Paterson IC, Choo SW. Comparative genome analysis of Fusobacterium nucleatum. Genome Biol Evol 2016;8:2928-2938.

38. Metzger Z, Lin Y-Y, Dimeo F, Ambrose WW, Trope M, Arnold RR. Synergistic pathogenicity of Porphyromonas gingivalis and Fusobacterium nucleatum in the mouse subcutaneous chamber model. J Endod 2009; 35:86-94

39. Heuer W, Elter C, Demling A, et al. Analysis of early biofilm formation on oral implants in man. $J$ Oral Rehabil 2007;34:377-382.

40. Cosgarea R, Dannewitz B, Sculean A, et al. Bacterial and inflammatory behavior of implants in the early healing phase of chronic periodontitis. Quintessence Int 2012;43:491-501.

41. Heuer W, Kettenring A, Stumpp SN, et al. Metagenomic analysis of the peri-implant and periodontal microflora 
in patients with clinical signs of gingivitis or mucositis. Clin Oral Investig 2012;16:843-850.

42. Vered Y, Zini A, Mann J, et al. Teeth and implant surroundings: Clinical health indices and microbiologic parameters. Quintessence Int 2011;42:339-344.

43. Cortelli SC, Cortelli JR, Romeiro RL, et al. Frequency of periodontal pathogens in equivalent peri-implant and periodontal clinical statuses. Arch Oral Biol 2013;58: 67-74.

44. Costalonga $M$, Herzberg $M C$. The oral microbiome and the immunobiology of periodontal disease and caries. Immunol Lett 2014;162(2 Pt A):22-38.

45. Yamanaka W, Takeshita T, Shibata Y, et al. Compositional stability of a salivary bacterial population against supragingival microbiota shift following periodontal therapy. PLoS One 2012;7:e42806.

46. van Winkelhoff AJ, van Steenbergen TJ, de Graaff J. Porphyromonas (Bacteroides) endodontalis: Its role in endodontal infections. J Endod 1992;18:431-434.

47. Rôças IN, Siqueira JF Jr. Prevalence of new candidate pathogens Prevotella baroniae, Prevotella multisaccharivorax and as-yet-uncultivated Bacteroidetes clone X083 in primary endodontic infections. J Endod 2009; 35:1359-1362.
48. Wu C. Human microbiome, Actinobacteria. In: Nelson KA, ed. Encyclopedia of Metagenomics. New York: Springer New York; 2013:1-7.

49. Socransky SS, Haffajee AD, Smith C, et al. Use of checkerboard DNA-DNA hybridization to study complex microbial ecosystems. Oral Microbiol Immunol 2004; 19:352-362.

50. van der Horst J, Buijs MJ, Laine ML, et al. Sterile paper points as a bacterial DNA-contamination source in microbiome profiles of clinical samples. J Dent 2013; 41:1297-1301.

51. Elter C, Heuer W, Demling A, et al. Supra- and subgingival biofilm formation on implant abutments with different surface characteristics. Int $J$ Oral Maxillofac Implants 2008;23:327-334.

Correspondence: Dr. Rui Figueiredo, Faculty of Medicine and Health Sciences, University of Barcelona, Bellvitge Campus, Pavello de Govern 2a planta, Despatx 2.9, L'Hospitalet de Llobregat, 08907 Barcelona, Spain. Email: ruipfigueiredo@hotmail.com.

Submitted January 21, 2017; accepted for publication April 28, 2017. 